\title{
Improving Interpretation, Presentation and Visualisation of LCA Studies for Decision Making Support
}

\author{
Serenella Sala and Jessica Andreasson
}

\begin{abstract}
Interpretation, presentation, and visualization of life cycle assessment (LCA) results are key steps for ensuring proper decision support. Despite the interpretation being a crucial step, it is often not performed in a systematic way. For example, sensitivity analysis, variability and uncertainties analyses, comparison with results coming from different disciplines and domains are not properly done. So far, numerous LCA studies have been published and are often used by decision makers (both in business and policy contexts) to support the identification of hotspots or for drawing conclusions from meta-reviews while missing a good interpretation. Moreover, improving current practices in the visualisation of the results may help both the interpretation and the communication of LCA by a broader audience.
\end{abstract}

\section{Introduction}

According to ISO 14040, interpretation is the phase of LCA in which the findings from the inventory analysis and the impact assessment are considered together. It should deliver results that are consistent with the defined goal and scope of the study and which reach conclusions, explain limitations and provide recommendations. ISO 14044 further specifies that interpretation comprises the following elements: (i) identification of the significant issues based on the results of the life cycle inventory (LCI) and life cycle impact assessment (LCIA) phases of LCA; (ii) completeness, sensitivity and consistency checks; (iii) conclusions, limitations, and recommendations.

Despite the interpretation is a crucial step for supporting decision making, this step is often not performed in a systematic way. For example, sensitivity analysis from the

\author{
S. Sala $(\bowtie)$ \\ European Commission, Joint Research Centre, Directorate D-Sustainable Resources, \\ Bio-Economy Unit, via Enrico Fermi 2749, 21027 Ispra, VA, Italy \\ e-mail: serenella.sala@ec.europa.eu \\ J. Andreasson \\ Volvo Car Corporation, Gothenburg, Sweden \\ (C) The Author(s) 2018 \\ E. Benetto et al. (eds.), Designing Sustainable Technologies, \\ Products and Policies, https://doi.org/10.1007/978-3-319-66981-6_37
}


inventory step (e.g. between different source of secondary data) [1] up to the impact assessment (e.g. between different methods for impact assessment [2,3] and normalisation references [4]), variability and uncertainties analyses, comparison with results coming from different disciplines and domains are not properly done. So far, numerous LCA studies have been published and are often used by decision makers (both in business and policy contexts) to support the identification of hotspots or for drawing conclusions from meta-reviews while missing a good interpretation. In 2016, a guidance on interpretation has been published by the European Commission, Joint Research Centre [5], aiming at fostering a discussion on the critical role of interpretation and visualization of results. Besides, very different audiences, having different backgrounds and expectations, may use LCA results: decision-makers, scientists, public authorities, consumers, etc. The way LCA results are presented is too often generic instead of being tailored to their needs. Moreover, LCA deals with numerous environmental impact indicators, large amount of data, uncertainties, spatial differentiation etc. LCA has evolved from a simple carbon and energy accounting to the assessment of large and complex consequential systems. Still, the way the results are presented has not changed a lot, i.e. mainly bar-charts, pie-charts, simple tables and Sankey diagrams. In the meantime, data visualization and analysis has evolved, with new interactive ways of displaying results, which may both help interpretation and communication of the results.

\section{Interpretation, Presentation and Visualisation}

The session on "Improving interpretation, presentation and visualisation of LCA studies for decision making support" at LCM 2017 aimed at discussing how to promote a transition toward more robust interpretation, presentation, and visualization of the LCA results. This includes the identification of key elements and criteria for knowledge capitalization, with regard to existing studies.

Life cycle-based methodologies as LCA but also other integrated assessment approaches are used in decision-making processes, product development and marketing activities. LCA methodology may be used as a methodology for supporting the identification of 'hot spots' in the value chain and measuring progress towards sustainability targets. It is important for companies to find common ground on how to implement these approaches, which data assessments and impact assessments to be used and how results should be interpreted. ISO rules give a good basis for that work, though is not sufficient.

\subsection{Identifying Target Audience for LCA Results}

Within a company or an institution, before any successful adaptation and visualization of life cycle information, it is important for internal life cycle experts to 
identify and understand how other functions of the company or institution perceive and value life cycle information in their specific working situations and decision contexts. To get a better understanding of these internal users of life cycle information, life cycle experts in four multinational companies (Akzo Nobel, Vattenfall, Volvo Cars, Volvo Group) have joined forces with researchers in Life Cycle Management and behavioural science to create a graphical map of how life cycle information is spread and used in different parts of an organization. The map is constructed by combining (a) inventories on how quantitative data seeks its way to internal users through databases, reports and Key Performance Indicators (KPIs), with (b) qualitative interviews on goal framing and decision weights of e.g. environmental and economic information. As a result, the map illustrates both the "physical" flows of life cycle information and the "cognitive logics" of this information for different users (e.g. how values, attitudes and norms influence the target groups' likelihood of including life cycle information in their decision processes). Based on the map, each company can identify and discuss who the main users of life cycle information are and what premises for life cycle thinking these users have. In what decision-making situation is, or can, life cycle information be used? How is the information understood? What other sources of information and rationales for decisions are used in parallel to, or in conflict with, LCA results? Initial analyses on the usefulness of the map point to a better understanding of how life cycle experts can tailor information for decisions in different parts of the company, as well as on its usefulness in illustrating to people outside of the environmental departments the widespread use of life cycle information that already exist in the company.

Moreover, LCA results may be used to influence design solution for products, needing an interaction between LCA experts, products designer and consumers. This often happens is a context with certain legislative constraints or requirement. A model for supporting life cycle design of food and packaging has been presented at the LMC conference. Packaging designer cannot give packaging all functions, because it is impossible to grasp all relations among demands and functions, and there are trade-off relations among them. An activity model visualizing information flows among stakeholders was constructed to design an environmentally conscious product, having a broad life cycle perspective. This allowed to optimise design parameters (e.g., packaging material and weight, filling rate) and functions (e.g., permeability, usability) along the life cycle of food products.

\subsection{Tailoring the Presentation of LCA Results and Promoting Best Practices}

Several challenges are faced by industrial sustainability experts and practitioners when communicating and visualising LCA results in different decision-making contexts. The project SAMT (Sustainability assessment methods and tools to support decision-making in the process industries) [6], was focusing on 
communication of LCA results in different decision-making contexts addressing all those challenges. Experiences and good practices from the cement, oil, metal, water, waste and chemical industry were collected using interviews, workshops and practical case studies. Crucial aspects identified during the project were, among others: (i) communicating uncertainty; (ii) explaining the proximate and underlying causes of variability in results; (iii) integrating results representing different impact categories and defining proper benchmarks for comparison. Challenges related to interpretation and communication are faced especially in situations in which a decrease in one indicator or impact category is offset with an increase in another category. The findings of the project indicate that many of the challenges related to communication are similar despite of the sector in question. There is also a need to harmonise the way results are presented, in order to increase transparency and improve comparability of the studies.

For exchanging experiences, updating or adopting methods, the "International Sustainability Practitioners Network" (ISPN) was created since 2012. The ISPN is an exchange forum for LCA methodology in the context of industry and comprises sustainability experts from a range of different industry sectors. Examples of good practices of this cross-sectoral initiative and opportunities for improving sustainability assessments within of companies are the focus of the exchange between experts. The ISPN members address challenges and solutions in terms of data availability and uncertainty, streamlining and using standardization processes as well as communication of results with non-LCA-experts.

\subsection{Improving Visualisation of LCA Results}

Given the perceived complexity of LCA, improving the presentation and visualisation of results and the uncertainties thereof is crucial for decision support. New features have been presented for two softwares, namely Open LCA [7] and RangeLCA [8].

OpenLCA has expanded its interpretation component in order to better present the hotspots analysis using new visualisation features from the open source: radar charts, sun burst graphs, contribution trees, geographical display of regionalized results, and tables with the values of inventory, impact results and impact factor per elementary flow contributing to the hotspot towards a structured interpretation of the results. Moreover, an assessment and effective handling of the data quality is also key for the interpretation especially if the study is used for decision support. OpenLCA proposes an innovative way to calculate, aggregate and display data quality information along the life cycle, to make the detection of data quality hotspots easier, i.e. to detect e.g. whether hotspots appear in the case study to some extent because of weak data quality, or whether hotspots call for immediate action because they are based on reliable information. The calculated data quality is shown along with the LCI and LCIA results in the different visualisations of openLCA, including impact analysis tables and Sankey diagram. 
Besides, the LCA software RangeLCA is proposing new ways of visualizing comparative LCA results for representing the diversity of individual cases and the uncertainty. RangeLCA, automatically integrates a full sensitivity analysis by associating probability distributions or discrete ranges of values to each variable parameter. LCIA results are calculated for a large number of cases, each corresponding to a possible combination of variable parameters. For each impact category, results of all cases are plotted as a "cloud of points" in function of the most influential parameters. When comparing two systems, common variable parameters take common values for a same case. Results can hence be compared two by two and a delta between systems can be calculated for each case. Plotting these delta values in "delta graphs" allows the common variability to be eliminated and the interpretation to concentrate on the remaining variability. A colour code distinguishes positive and negative deltas. These graphs allow identifying areas of relevance where conclusions can be drawn for certain ranges of values of two main parameters. This type of graphs allows acquiring a more complete vision of a complex system, reliability of conclusions in comparative LCA by showing whether differences are significant or not in terms of sign and amplitude. Delta graphs and areas of relevance help LCA studies to answer much broader questions, such as: "In which range of situations is a system rather better than another one? "

\section{Conclusion and Outlook}

The last phase of a LCA, namely the interpretation of the life cycle inventory and impact assessment results, is especially important in LCM as it is key for decision-making and communication of results. Ability to create meaningful and easily understandable results would be essential in order to increase the acceptability and applicability of the methods, and to mainstream their use in different decision-making situations, that may include for example product and process development, supply chain management, investment decisions, marketing and responding to stakeholder requests. Future effort should be both on the side of improving the analytical power of LCA tools adopted by practitioners and in the understanding of the diversified communication needs of a wide range of stakeholders both in business and institutional context. From the analytical aspects point of view, a systematic approach in the interpretation and hotspots analyses, including methods for sensitivity analysis, variability and uncertainty analysis, is needed. From the communication point of view, there is the need of improving the identification of the target audience of the results, for tailoring the communication, including the rationale on how to choose visualization and graphical tools depending on the key messages to show and on the audience. This could be facilitated by the adoption of new ways of visualizing LCA results, using interactive and advanced visualization tools for both external and internal stakeholders. 


\section{References}

1. Corrado S, Zampori L, Castellani V, Sala S, Systematic analysis of secondary Life Cycle Inventories when modelling agricultural production: a case study for arable crops, Journal of Cleaner Production, Vol. 172, 2018, pp. 3990-4000.

2. Rigamonti L, Falbo A, Zampori L, Sala S, Supporting a transition towards sustainable circular economy: sensitivity analysis for the interpretation of LCA for the recovery of electric and electronic waste, International Journal of Life Cycle Assessment, Vol. 22, No. 8, 2017, pp. $1278-1287$.

3. Castellani V, Lorenzo B, Sala S, Hotspots analysis and critical interpretation of food life cycle assessment studies for selecting eco-innovation options and for policy support, Journal of Cleaner Production, Vol. 140, No. 2, 2017, pp. 556-568.

4. Benini L, Sala S, Integrated assessment of environmental impact of Europe in 2010: uncertainty and sensitivity of the normalisation factors to methodological assumptions, International Journal of Life Cycle Assessment, Vol. 21, No. 2, 2016, pp. 224-236.

5. Zampori L, Saouter E, Schau E, Cristobal Garcia J, Castellani V, Sala S, Guide for interpreting life cycle assessment result, JRC technical report, Publications Office of the European Union; 2016.

6. https://www.spire2030.eu/samt, (Accessed 05.09.2017).

7. http://www.openlca.org/, (Accessed 05.09.2017).

8. http://www.rdcenvironment.be, (Accessed 05.09.2017).

Open Access This chapter is licensed under the terms of the Creative Commons Attribution 4.0 International License (http://creativecommons.org/licenses/by/4.0/), which permits use, sharing, adaptation, distribution and reproduction in any medium or format, as long as you give appropriate credit to the original author(s) and the source, provide a link to the Creative Commons license and indicate if changes were made.

The images or other third party material in this chapter are included in the chapter's Creative Commons license, unless indicated otherwise in a credit line to the material. If material is not included in the chapter's Creative Commons license and your intended use is not permitted by statutory regulation or exceeds the permitted use, you will need to obtain permission directly from the copyright holder. 